\title{
Neuroendoscopys way in the treatment of posterior fossa tumours associated hydrocephalus in children
}

\begin{abstract}
Background: Brain neuroendoscopy has been introduced to our neurosurgical department since January 2000. Neuroendoscope was used to treat hydrocephalus secondary to posterior fossa tumours. In this study we expand on our previously published data to reassess and present our experience over the last sixteen years.

Objective: This article condenses our added -to previous paper- long-term experience with neuroendoscopy in the Treatment of obstructive Hydrocephalus associated with posterior fossa tumors in Children, highlighting the prominent challenges and outcomes.

Methods: A retrospective study conducted to assess our patients operated between January 2000 and January 2016, 202 children with posterior fossa tumor were managed. One Hundred eighty-one patients had symptomatic hydrocephalus. The other 21 cases had no hydrocephalus or non-symptomatic mild dilatation of ventricles, they were excluded from the study. There were 87 males and 94 females, mean age: 5.49 \pm 3.2 ; (range: $2-14$ ). Mean follow-up $32 \pm 7$ months (range 13-156). Endoscopic third ventriculostomy was performed in all cases to relieve intracranial pressure as an urgent procedure after admission.

Results:Endoscopic third ventriculostomy in principle is efficacious, the procedure resolves the increased intracranial pressure before posterior fossa surgery in all cases, reduces risk of post operative hydrocephalus, eliminate the risk of upward herniation, and provides a burr hole that can be used as an emergency portal for CSF drainage. Four cases were complicated with infection, two cases of CSF leak. Ten cases developed post- operative hydrocephalus and were treated by VP shunt insertion.

Conclusion:Endoscopic third ventriculostomy is a rapidly evolving field in the management of hydrocephalus secondary to posterior fossa tumors due to its simplicity, minimal invasive, the measurable good outcome and high success rate. It is a feasible option for the emergency control of severe hydrocephalus caused by posterior fossa tumors; it eliminates reliance on mechanical shunts with all their limitations and complications. It re establishes CSF dynamics to essentially normal status eliminating the risk of upward herniation.
\end{abstract}

Keywords: hydrocephalus, endoscopic third ventriculostomy, neuroendoscopy
Volume 6 Issue 3 - 2017

\author{
Al Qroom Rami, Khasawneh Nidal, Haddad \\ Feras, Alqurashi Muath \\ Department of Neurosurgery, King Hussein Medical Centre, \\ Jordan
}

Correspondence: Al Qroom Rami, Department of Neurosurgery, King Hussein Medical Centre, Amman, Jordan, Email dr.alqroomrami@gmail.com

Received: January 27, 2017 | Published: March 16, 2017

\section{Introduction}

Brain tumors are of the most devastating forms of human disorders, particularly when they arise in the posterior fossa. The proximity of these lesions to the brainstem and fourth ventricle explains the common presentation of these patients. Obstructive hydrocephalus is described in about $80 \%$ of the cases, it can lead to herniation and death if left untreated. ${ }^{1-7}$

The best way to handle obstructive hydrocephalus secondary to posterior fossa tumor is still subject to debate. Approaches include preoperative insertion of a cerebrospinal fluid shunt or external ventricular drainage followed by surgical resection of the tumor. ${ }^{1,2,4,6-9}$ Others recommend treatment with corticosteroids and direct approach to the posterior fossa pathology, when possible. ${ }^{10-17}$

Endoscopy capitulates on improved visualization and permits effective surgical therapy of lesions in the nadirs of the brain with minimal collateral interruption of eloquent parenchyma. It is currently established popularity emulates the diversity of increasing applications, which are also reflective of integral challenges. Based on recent reports. ${ }^{6,713,18,19}$ Neuroendoscopic treatment of hydrocephalus (endoscopic third ventriculostomy) is the most recent approach for management. In our center, we adopted the policy of performing a preoperative endoscopic third ventriculostomy (ETV) in cases of symptomatic hydrocephalus.

\section{Methods}

A single institution, retrospective analysis of consecutive cases over a 16 year period (January 2000 to January 2016) carried out. Clinical records, radiological studies, operation notes as well as follow up records, were obtained and analyzed. 202 patients with posterior fossa tumor were admitted to the neurosurgery department at King Hussein Medical Centre. All 202 patients had a CT scan upon admission \pm magnetic resonance imaging (MRI). Patients with no hydrocephalus, mild non-symptomatic dilatation of ventricles or previously shunted patients were excluded from this study. One hundred eighty-one patients with radiological evidence of severe hydrocephalus and raised intracranial pressure constituted the study group. Patients' age ranged from 2 years to 14 years, mean age: 5.49 \pm 3.2 ; (range: 2-14), 87 males and 94 females, All patients were started on corticosteroids on admission. Brain and whole spine MRI were obtained when possible and ETV performed on an urgent basis (17 cases as an emergency procedure). Follow up CT scan was performed after ETV for all patients.

\section{Surgical details}

The patient is placed in supine position with the head slightly flexed. A pre-coronal burr hole is performed with the optimal entry position at $3 \mathrm{~cm}$ lateral to the midline and $1 \mathrm{~cm}$ anterior to the coronal suture (Figure 1). The rigid endoscope is advanced into the lateral 
ventricle. Under direct vision, the endoscope is passed through the foramen of Monro into the third ventricle- the foramen of Monro can be identified by the thalamostriate vein and choroid plexus (Figure $2)$. The third ventricle is inspected prior to perforation of the floor. The ventriculostomy is placed just anterior to the mammillary bodies. Perforation is done with blunt probe followed by balloon catheter dilatation (Figures 3). Entry into the prepontine cistern is performed with caution so as to avoid injury to the basilar apex and perforating vessels. Hemostasis -if needed- with irrigation is achieved until a clear operative field is visualized. Tumour resection is scheduled under non-emergency conditions on the next available or convenient list.

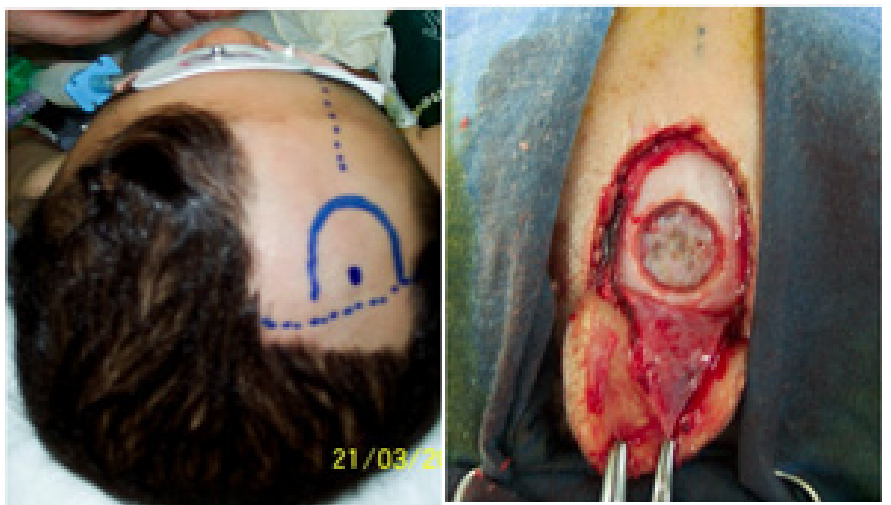

Figure I $\mathrm{N}=57$; Epidemiological distribution of the pathological fractures, traumatic fractures, and nonunion.

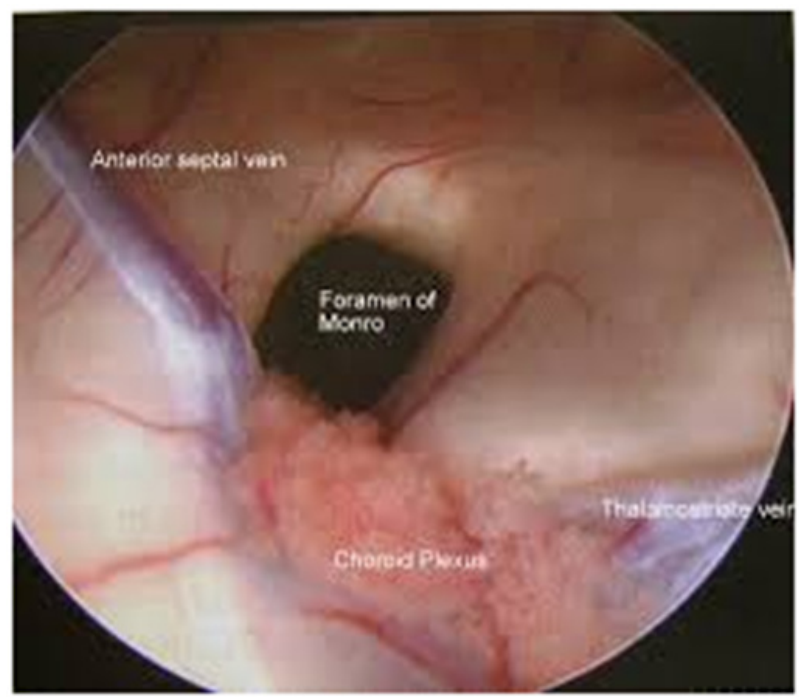

Figure $2 \mathrm{~N}=57$; Epidemiological distribution of the pathological fractures, traumatic fractures, and nonunion.
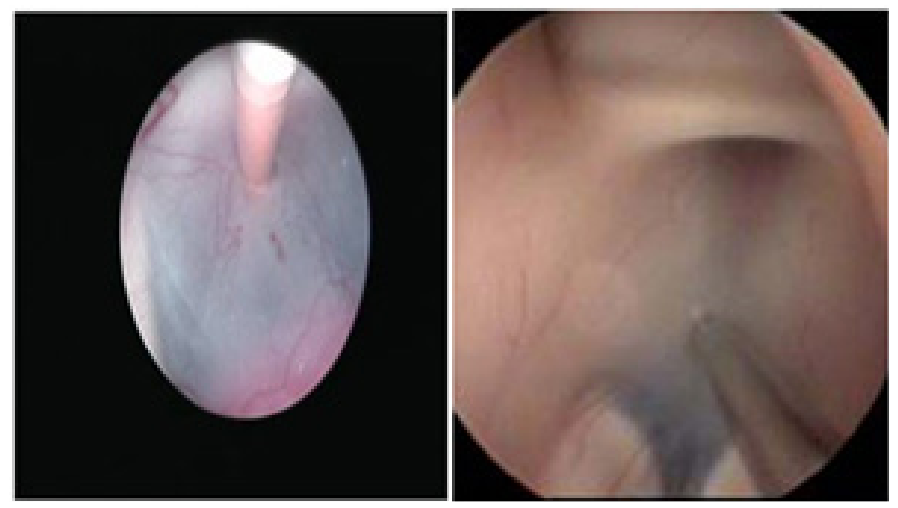
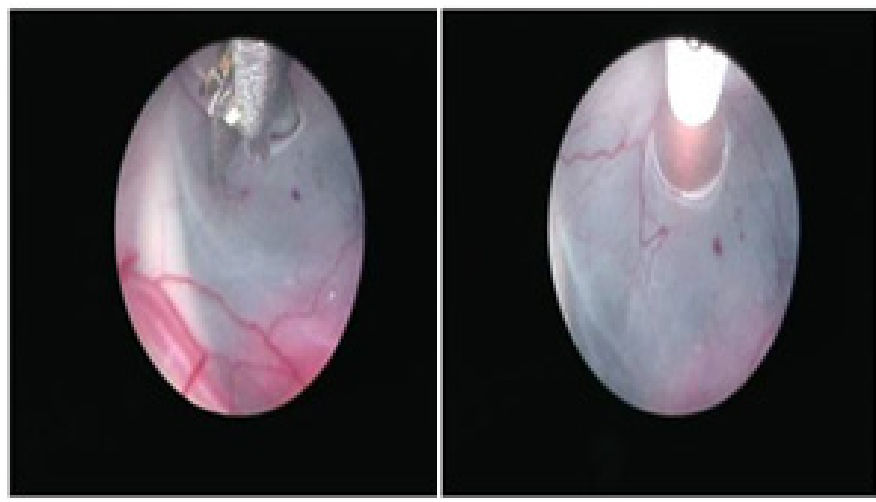

Figure $3 \mathrm{~N}=57$; Epidemiological distribution of the pathological fractures, traumatic fractures, and nonunion.

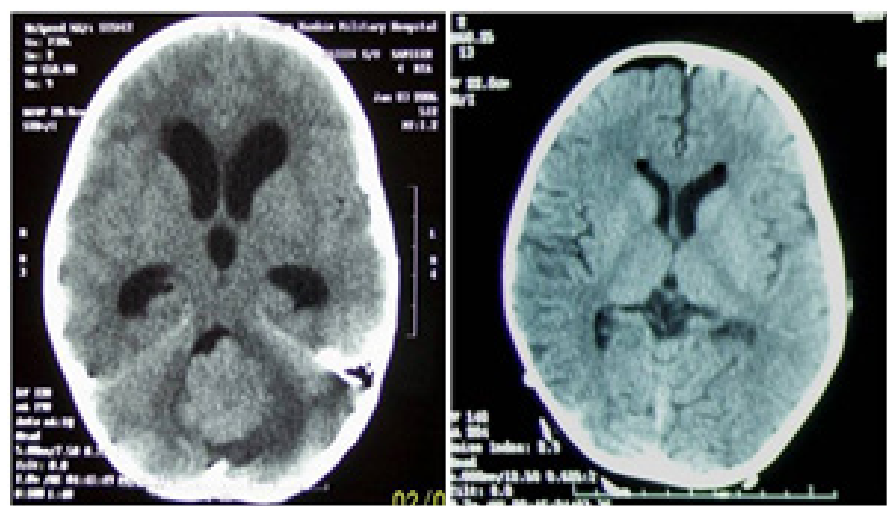

Figure $4 \mathrm{~N}=57$; Epidemiological distribution of the pathological fractures, traumatic fractures, and nonunion.

\section{Results}

Endoscopic third ventriculostomy was performed in the 181 patients. There were no technical difficulties in the procedure even in the cases associated with anatomical distortion of the floor of the third ventricle due to the tumor. Ventricular drainage device (reservoir) was inserted in 13 cases for the suspicion of inadequate ventriculostomy. The signs and symptoms of increased intracranial pressure resolved after ETV in all patients. Follow-up brain CT scan showed no complications related to the third ventriculostomy except for insignificant asymptomatic Pneumocephalus in 11 cases. The size of ventricles showed a reduction in 168 cases (93\%) (Figure 4).

Four patients developed fever and meningeal irritation signs and symptoms after 3 days from ETV, the external ventricular drain was inserted and antibiotics started for one week. The patient improved and surgery was performed with no sequels, patients maintained on antibiotics for another week after surgery. One hundred sixty- two cases underwent definitive surgery on the next available operating list that is 2-4 days from performing ETV. Surgery in 14 patients was deferred till the general and clinical conditions improved, those patients presented with an altered level of consciousness and poor general condition related to vomiting and increased intracranial pressure. Five patients' operation were delayed due to theater time availability.

The definitive surgery showed satisfactory posterior fossa condition in relation to the CSF pressure. Total resection was achieved in all cases except for 7 cases where the tumor was adherent to the floor of the fourth ventricle. The anatomical pathway of the CSF was 
opened in all cases with a satisfactory flow. The post-tumor resection period showed no complications related to CSF pressure; there was no hydrocephalus, no CSF leak, or CSF collection in the wound area in all cases. ETV proved successful in the immediate and longterm control of hydrocephalus. There was no deaths nor permanent morbidity related to the procedure. On Long-term follow-up 10 patients developed symptomatic hydrocephalus which was treated with Venticulo-peritoneal shunt.

\section{Discussion}

Neuro-oncology provides an ideal arena for the application of endoscopy. The advantages of improved visualization of intraventricular pathology, advanced management of tumor-related hydrocephalus, and minimally invasive biopsies and, or removal of intraventricular tumors are precious enhancements to modern tumor management. The association of posterior fossa tumors with hydrocephalus, both potentially fatal conditions, imposes urgent surgical treatment. The routine placement of preoperative shunts significantly reduces the overall morbidity and mortality rates. ${ }^{1}$

Nevertheless, several arguments have been raised against systematic pre-shunting, depending on the reported $10 \%$ rate of upward herniation in cases of posterior fossa tumors subjected to preliminary shunting, and spreading of medulloblastomas through ventriculoperitoneal shunts. ${ }^{1,15-17}$ These arguments and the improvements in the availability and type of neuroimaging systems that permit earlier diagnosis have caused neurosurgeons to question the need for routine shunt placement.

The revival of neuroendoscopy can be traced to advancement in optics and computer technology. ${ }^{18}$ Endoscopic third ventriculostomy in the management of hydrocephalus secondary to posterior fossa tumors was proposed, for the first time, by Chumas et al., ${ }^{9}$ in 1995 and its efficacy was reviewed by Sainte-Rose et al., ${ }^{19}$ in 2001. ETV creates a communication between the ventricular system and subarachnoid spaces at the level of the floor of the third ventricle.

Preliminary ETV allowed time to resuscitate patients, arrange for proper neuroimaging and schedule the patients for definitive surgery on a more convenient basis. ETV resulted in satisfactory posterior fossa surgical conditions. ETV has been shown to offer an acceptable low cost alternative treatment to shunts especially in children with obstructive hydrocephalus in developing countries. ${ }^{20,21}$ There are numerous studies now confirming the high success rate and low complication rate of ETV. It is now considered a safe and effective treatment for obstructive hydrocephalus in selected patients. ${ }^{22,23}$ Indications for performing ETV are based on computed tomography or magnetic resonance imaging (MRI) findings that demonstrate a noncommunicating type hydrocephalus with obstruction at the level of, or distal to, the posterior third ventricle. Patients with hydrocephalus from aqueductal stenosis are, in general, excellent candidates for ETV.

Although controversial, patients less than 6 months of age have not shown uniformly good results with ETV, and most authors do not advocate the procedure in this group. ${ }^{24}$ ETV has a role in the treatment of hydrocephalus secondary to posterior fossa tumors and is being used for that application in many centers..$^{25}$ Sainte-Rose et al., ${ }^{19}$ reviewed 67 ETVs performed before tumor removal in patients with severe hydrocephalus. In this series, there were no deaths and no permanent morbidity related to the procedure, a $98.5 \%$ rate of immediate symptomatic resolution, and a $94 \%$ rate of shunt-free patients after tumor removal. ${ }^{19}$ Comparing these results with patients with hydrocephalus who underwent a "conventional treatment" (steroid medications, early surgery, and ventricular drainage) and with patients with no evidence of ventricular enlargement, they concluded that ETV had a curative effect on intracranial hypertension and a prophylactic effect by preventing the development of hydrocephalus after tumour removal. Our results were comparable to this study we had a rate of $93 \%$ shunt free cases in long-term follow-up. In our current study, we have found a more positive response with ETV for relieving of increased intracranial pressure.

The difference between our previous report and our current study ( $93 \%$ vs. $83 \%$ ), may partly have resulted from the small sample size and mid-term follow-up in comparison to our current experience.

Pre-craniotomy normalization of CSF hydrodynamics seems to decrease the risk of permanent postoperative impairment of CSF circulation. Hopf et al., ${ }^{26} \&$ Valenzuela and Trellez ${ }^{27}$ have also reported a significant experience in 17 and 21 cases respectively, both with a $76 \%$ success rate in controlling hydrocephalus.

This study presents our current practice and highlights the challenges we experienced in performing endoscopic surgery in our center. We adopted the policy of treating severe hydrocephalus in patients with posterior fossa tumor with ETV. The patients were started as well on steroids on admission. The procedure seems to provide a valid alternative to placement of a permanent shunt in cases in which hydrocephalus develops following posterior fossa tumor. This policy had to surpass a myriad of obstacles which interface the successful set up of neuroendoscopy service including: The infrastructure and the equipment required to perform such procedure, the Steep training curve on the basic principles and practice of neuroendoscopy, which we incapacitated by multidisciplinary team approach, practice on models, cadaveric dissection and attending live operative workshops.

ETV has numerous potential benefits over the standard shunt procedure, which possesses its own set of inherent risks and complications. During neuroendoscopy more often reported events are: Injury to the fornix, intraventricular hemorrhage, basilar artery injury, cardiac arrhythmias or respiratory arrest and failed ETV. ${ }^{28-30}$ Our study reported the following complications: there were 4 cases of fever and meningeal irritation. In 2 patients CSF leakage developed through the burr hole incision site; treated with a pressure dressing. Our work supports the trend adopting the preoperative ETV for the management of all cases of symptomatic hydrocephalus secondary to posterior fossa tumors and in accordance with the work other authors adopting the same policy concluded. ${ }^{31-34}$

\section{Conclusion}

Neuroendoscopy is a rapidly evolving field which adheres to the principles of minimally invasive surgery that can be performed on an emergency basis, allowing rapid relief of clinical signs and symptoms of raised intracranial pressure due to hydrocephalus secondary to posterior fossa tumors. Eliminates reliance on mechanical shunts with all their limitations, and returns CSF dynamics to essentially normal physiological status in patients with obstructive hydrocephalus.

The indications for this technique of neuroendoscopy are swiftly expanding. The burr hole used for ETV can provide a rapid access to the ventricles post-operatively if the need arises. CSF is obtained for cytology during the procedure. Despite myriad obstacles which interface the successful set up of neuroendoscopy service. ETV proved successful in the immediate and long-term control of hydrocephalus both clinically and radiologically with minimal risk and complications. 


\section{Acknowledgments}

None.

\section{Conflicts of interest}

None.

\section{Funding}

None.

\section{References}

1. Khasawneh N, Bani Mustafa H, Abu Wandi E. Endoscopic Third Ventriculostomy in the Treatment of Hydrocephalus Secondary to Posterior Fossa Tumours In Children. JRMS. 2008; 15(2):47-51.

2. Albright AL. The value of pre-craniotomy shunts in children with posterior fossa tumors. Clin Neurosurg. 1983;30:278-285.

3. Albright L, Reigel DH. Management of hydrocephalus secondary to posterior fossa tumors. J Neurosurg. 1977;46:52-55.

4. Bognar L, Borgulya G, Benke P, et al. Analysis of CSF shunting procedures requirement in children with posterior fossa tumors. Childs Nerv Syst. 2003;19(5-6):332-336.

5. B hm B, Mohadjer M, Hemmer R. Preoperative continuous measurements of ventricular pressure in hydrocephalus occlusus with tumors of the posterior fossa: the value of ventriculoatrial shunt. $A d v$ Neurosurg. 1978;5:194-198

6. Cassoto A, Buoncristiani P. Medulloblastoma in childhood. Childs Brain. 1982;9(3-4):299-308.

7. Lee $\mathrm{M}$, Wisoff $\mathrm{JH}$, Abbott R, et al. Management of hydrocephalus in children with medulloblastomas: prognostic factors for shunting. Pediatr Neurosurg. 1994;20(4):240-247.

8. Richard NE. Long-term measurement of ventricular CSF pressure with tumors of the posterior fossa. Adv Neurosurg. 1978;5:179-187.

9. Chumas P, Saint Rose C, Cinalli G. III Ventriculostomy in the management of posterior fossa tumors in children. Childs Nerv Syst. $1995 ; 11: 540$

10. Cinalli G. Alternatives to shunting. Childs Nerv Syst. 1999;15(11):718-731.

11. Culley DJ, Berger MS, Shaw D, et al. An analysis of factors determining the need for ventriculoperitoneal shunts after posterior fossa tumo surgery in children. Clinical study. Neurosurgery. 1994;34(3):402-408.

12. Gross P, Goat M, Knoblich OE. Disorders of CSF circulation after interventions in the area of the posterior cranial fossa with prior shunt operation. Adv Neurosurg. 1978;5:199-202.

13. Papo I, Caruselli G, Luongo A. External ventricular drainage in the management of posterior fossa tumors in children and adolescents. Neurosurgery. 1982;10:13-15.

14. Rappaport ZH, Shalit MN. Perioperative external drainage in obstructive hydrocephalus secondary to infratentorial brain tumors. Acta Neurochir. 1989;96(3):118-121.

15. Epstein F, Murali R. Paediatric posterior fossa tumors: hazards of the "preoperative" shunt. Neurosurgery. 1978;3(3):348-350.
16. Fiorillo A, Maggi G, Martone A, et al. Shunt-related abdominal metastases in an infant with medulloblastoma: long-term remission by systemic chemotherapy and surgery. J Neurooncol. 2001;52(3):273-276.

17. Hoffman HJ, Hendrick EB, Humphreys RP. Metastasis via ventriculoperitoneal shunt in patients with medulloblastoma. $J$ Neurosurg. 1976;44(5):562-566.

18. Griffith HB. Technique of fontanelle and persutural ventriculoscopy and endoscopic ventricular surgery in infants. Childs Brain. 1975;1(6):359-363.

19. Sainte Rose C, Cinalli G, Roux FE, et al. Management of hydrocephalus in pediatric patients with posterior fossa tumors: the role of endoscopic third ventriculostomy. J Neurosurg. 2001;95(5):791-797.

20. Jiménez León JC, Jiménez CS, Betancourt YM. Neuroendoscopy. Its usefulness in the hydrocephalus management of children in developing countries. Medicina (B Aires). 2007;67(6 Pt 1):665-673.

21. Warf BC, Tracy S, Mugamba J. Longterm outcome for endoscopic third ventriculostomy alone or in combination with choroid plexus cauterization or congenital aqueductal stenosis in African infants. $J$ Neurosurg Pediatr. 2012;10(2):108-111.

22. Hopf N, Grunert P, Fries G, et al. Endoscopic third ventriculostomy: Outcome analysis of 100 consecutive procedures. Neurosurgery. 1999;44(4):795-804.

23. Buxton N, Ho KJ, Macarthur D, et al. Neuroendoscopic third ventriculostomy for hydrocephalus in adults: report of a single unit's experience with 63 cases. Surg Neurol. 2001;55(2):74-78.

24. Cinalli G, Saint Rose C, Chumas P, et al. Failure of third ventriculostomy in the treatment of aqueductal stenosis in children. $J$ Neurosurg. 1999;90(3):448-454

25. Teo C, Young R. Endoscopic management of hydrocephalus secondary to posterior third ventricular tumors. Neurosurg Focus. 1999;7(4):e2.

26. Valenzuela S, Trellez A. Pediatric neuroendoscopy in Chile. Analysis of the first 100 cases. Childs Nerv Syst. 1999;15(9):457-460.

27. Yadav YR, Shenoy R, Mukerji G, et al. Water jet dissection technique for endoscopic third ventriculostomy minimises the risk of bleeding and neurological complications in obstructive hydrocephalus with a thick and opaque third ventricle floor. Minimally invasive neurosurgery (MIN). 2010;53(4):155-158.

28. Yadav YR, Parihar V, Pande S, et al. Endoscopic third ventriculostomy. Journal of neurosciences in rural practice. 2012;3(2):163-173.

29. Yadav Y, Parihar V, Kher Y. Complication avoidance and its management in endoscopic neurosurgery. Neurol India. 2013;61(3):217-225.

30. Muszynski CA, Laurent JP, Cheek WR. Effect of ventricular drainage and dural closure on cerebro-spinal fluid leaks after posterior fossa tumor surgery. Pediatr Neurosurg. 1994;21(4):227-231.

31. Schmid UD, Seiler RW. Management of obstructive hydrocephalus secondary to posterior fossa tumors by steroids and subcutaneous ventricular catheter reservoir. J Neurosurg. 1986;65(5):649-653.

32. Shalit MN, Ben Ari Y, Eynan N. The management of obstructive hydrocephalus by the use of external continuous ventricular drainage. Acta Neurochir (Wien). 1979;47(3-4):161-172.

33. Taylor WAS, Todd NV, Leighton SEJ. CSF drainage in patients with posterior fossa tumors. Acta Neurochir (Wien). 1992;117(1):1-6. 